\title{
Magnetic Resonance Neurographic Findings in Classic Idiopathic Neuralgic Amyotrophy in Subacute Stage: A Report of Four Cases
}

\author{
Myung Seok Park, MD ${ }^{1}$, Du Hwan Kim, MD², Duk Hyun Sung, MD ${ }^{1}$
}

\begin{abstract}
${ }^{1}$ Department of Physical and Rehabilitation Medicine, Samsung Medical Center, Sungkyunkwan University School of Medicine, Seoul; ${ }^{2}$ Department of Rehabilitation Medicine, Dongsan Medical Center, Keimyung University School of Medicine, Daegu, Korea
\end{abstract}

Neuralgic amyotrophy (NA) is characterized by acute onset of severe pain, followed by muscular weakness and wasting of the shoulder girdle. While the diagnosis of NA mainly relies on the clinical history and examination, some investigations including electrophysiologic study and radiologic study may help to confirm the diagnosis. Magnetic resonance neurography (MRN), a new technique for the evaluation of peripheral nerve disorders, can be helpful in the diagnosis of NA. MRN presents additional benefits in comparison to conventional magnetic resonance imaging in the diagnosis of idiopathic NA (INA). In this report, we present the first four cases of classic INA diagnosed with MRN in subacute stage. MRN imaging modality should be considered in patients clinically suspected of INA.

Keywords Neuralgic amyotrophy, Idiopathic brachial plexus neuritis, Magnetic resonance neurography

\section{INTRODUCTION}

Neuralgic amyotrophy (NA), also known as ParsonageTurner syndrome, idiopathic brachial plexus neuritis, and acute brachial plexitis, is characterized by acute onset of extreme pain, followed by muscular weakness

Received August 2, 2013; Accepted August 29, 2013

Corresponding author: Duk Hyun Sung

Department of Physical and Rehabilitation Medicine, Samsung Medical Center, Sungkyunkwan University School of Medicine, 81 Irwon-ro, Gangnam-gu, Seoul 135-710, Korea

Tel: +82-2-3410-2813, Fax: +82-2-3410-2820, E-mail: yays.sung@samsung. com

(c) This is an open-access article distributed under the terms of the Creative Commons Attribution Non-Commercial License (http://creativecommons. org/licenses/by-nc/3.0) which permits unrestricted noncommercial use distribution, and reproduction in any medium, provided the original work is properly cited.

Copyright (C) 2014 by Korean Academy of Rehabilitation Medicine and wasting of the shoulder girdle $[1,2]$. The upper trunk of the brachial plexus is most commonly involved, but any other part of plexus or peripheral nerve, such as the anterior interosseous nerve or even lumbar plexus, can be affected [3]. The frequent association of attack with preceding infection and immunizations indicates an autoimmune pathogenesis, but the precise mechanism is unknown [2,3]. NA can occur as an autosomal dominant hereditary trait, known as hereditary neuralgic amyotrophy (HNA), which is caused by mutations in the gene septin 9 on chromosome 17q25. It also exists as a sporadic disorder (idiopathic NA [INA]) [2]. NA is primarily a clinical diagnosis, and no currently available test can confirm or exclude NA.

Although the diagnosis of NA mainly depends on the typical clinical presentation, recent reports have revealed that magnetic resonance neurography (MRN), 
a new technique for the evaluation of peripheral nerve disorders, may be helpful in the diagnosis of NA. MRN is seen to present more benefits compared to conventional magnetic resonance imaging (MRI) in the diagnosis of NA $[4,5]$. There are only two published reports of NA diagnosed with MRN, with one patient in the chronic stage and the other in the acute stage. To our knowledge, there is no published report for classic INA diagnosed with MRN in the subacute stage, 1-3 months after symptom onset. Here we report the first four cases of INA diagnosed with MRN in the subacute stage. All of the cases are "classic" INA, which has pain and subsequent weakness localized to the unilateral or bilateral shoulder and/ or arm with monophasic course [6].

\section{CASE REPORTS}

\section{Case 1}

A 52-year-old woman complained of severe pain in right upper arm for five days. The degree of pain was too severe for usual activities of daily life, and she could not rotate or extend her neck. Four days later, the severe pain was slightly decreased. On the fifth day from the pain onset, she realized that she could not move her arm upward. Two weeks later, she came to our clinic for the residual pain of the right upper arm and worsening of the right shoulder weakness. According to the Medical Research Council (MRC) grade, there was $2 / 5$ strength of the right shoulder abductor and flexor, and $4 / 5$ of the right elbow flexor. There was no other weakness in the distal forearm or hand intrinsic muscle. Deep tendon reflex (DTR) test and sensory examinations including pin prick and light touch did not reveal significant difference compared to the other side.

Electrodiagnostic tests were performed at one month from the onset. Sensory nerve conduction studies (NCSs) including the median, ulnar, medial/lateral antebrachial cutaneous (MABC/LABC) nerves did not show any abnormal findings. Motor NCSs were also recorded in the median, ulnar, musculocutaneous, axillary and suprascapular nerves. Amplitude of compound muscle action potential was reduced to below half of the unaffected side in axillary and suprascapular nerve recordings. The needle electromyography revealed positive sharp waves and fibrillation potentials in right supraspinatus, infraspinatus, deltoid and biceps brachii. A radiologist (a specialist in the musculoskeletal system) checked her cervical spine MRI and confirmed that there were no findings related to disc herniation or radiculopathy.

The patient underwent MRN at one month from the onset of the symptoms (Fig. 1). MRN findings of the brachial plexus were collected, using a 3.0-Tesla scanner (Ingenia; Philips Medical Systems, Cleveland, OH, USA). Brachial plexus MRN employing coronal short-tau inversion recovery (STIR) images were obtained at TR/TE 5753/70. Diffusion-weighted (DW) MR neurographic images were also obtained at TR/TE 5411/80, in addition to gadolinium (Gd)-enhanced T1-weighted images. A high signal intensity (HSI) lesion in T2 STIR images and DW MR neurographic images and enhancement in T1 Gd enhancement images were shown from the postganglionic C5 and C6 root to the level of cord. The radiologist who

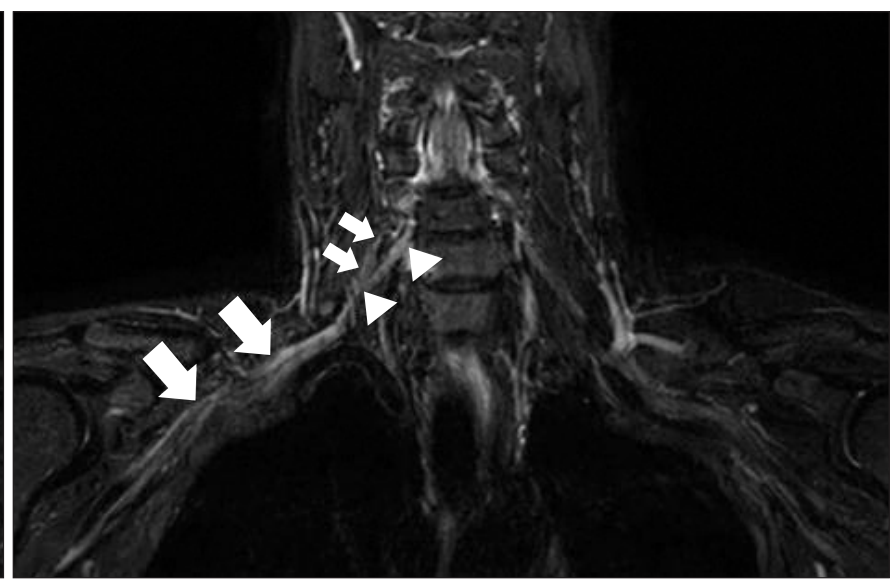

Fig. 1. The high signal intensity from the postganglionic right C5 (small arrows) and C6 (arrowheads) root to the level of cord (large arrows) in the coronal T2 short-tau inversion recovery image. 
checked MRI of the cervical spine reviewed the brachial plexus images without clinical information.

\section{Case 2}

A 73-year-old woman had a severe pain on the right scapular area. According to the numerical rating scale (NRS), the severity of the pain was 8-9/10. This severe right scapular pain was prolonged for one week, and then the severity was gradually decreased. Three weeks after the symptom onset, she had difficulty in elevating her right arm and could not comb her hair or wash her face. On physical examination, there was $2 / 5$ strength of the right shoulder abductor and flexor and $4 / 5$ strength of the right elbow flexor. Pin prick test did not show any difference between the two upper extremities. DTRs on the right biceps brachii and the right triceps brachii were absent. Electrophysiologic study performed at seven weeks from the onset was compatible with right brachial plexopathy, mainly involving upper trunk. Brachial plexus MRN was performed at seven weeks from the pain onset (Fig. 2A, B). Using the same machine as described in Case 1, coronal STIR images were obtained at TR/TE 5765/70. DW MR neurographic images and Gd-enhanced T1-weighted images were also obtained according to the same method as in Case 1. An HSI lesion extended from the postganglionic right $\mathrm{C} 5$ and $\mathrm{C} 6$ root to the level of cord in the T2 STIR images and in DW MR neurographic images. This lesion showed mild enhancement in Gdenhanced T1-weighted image.

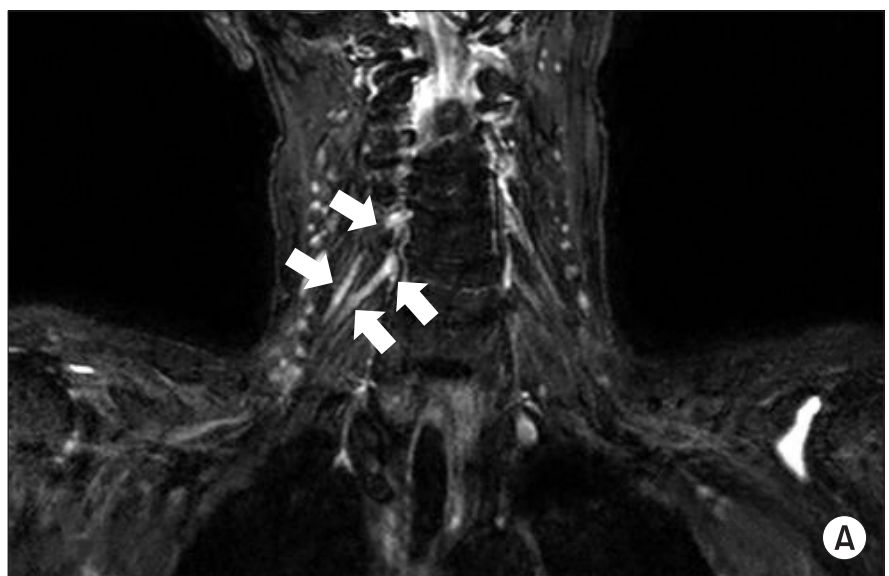

\section{Case 3}

A 65-year-old man felt a burning pain around his right periscapular area. The initial severity of pain was $5 / 10$ of NRS, and then the pain was slowly disappeared. After 2-3 weeks from the pain onset, he experienced weakness of the right shoulder and upper arm. Ten weeks from the pain onset, the patient was admitted to the Department of Neurosurgery at our hospital for surgical management of cervical spine. Before the surgical management, the surgeon suspected NA based on the characteristic clinical history. Moreover, mild stenosis of central canal in the level of C5-6 shown in MRI could not explain the clinical presentation of the patient. Motor weakness was shown in right shoulder abductor, shoulder flexor and elbow flexor as 4/5 strength of MRC grade. Sense of the right upper extremity was intact in pin prick test. Right biceps brachii had a decreased tendon reflex as compared to the unaffected side. Collective findings of NCS and needle electromyography were compatible with right brachial plexopathy involving upper trunk.

Eleven weeks after the symptom onset, the patient underwent brachial plexus MRN (Fig. 3). Coronal STIR images were acquired according to the method described in Case 2, using the same machine as used in Case 1. DW MR neurographic images were also obtained. The right C5 and C6 root were thickened and had increased signal intensity in T2 STIR images. The signal also increased from the right C5 and C6 root to the trunk in DW MR neurographic image. Gd-enhanced images failed to show definite enhancement of the nerve sheath.

Fig. 2. (A) The high signal intensity from the postganglionic right $\mathrm{C} 5$ and $\mathrm{C} 6$ root (small arrows) in the coronal T2 short-tau inversion recovery image. (B) The increased signal from the right C5 and C6 root (small arrows) to the level of cord (large arrow) in diffusion-weighted magnetic resonance neurography. 


\section{Case 4}

A 29-year-old man experienced severe pain around the left periscapular area and posterior neck. Due to the severity of the pain, he could not move his neck at all. The severe pain lasted for one week, and the intensity of pain was gradually decreased. On the fourth day from the symptom onset, he could not elevate his left upper extremity. In manual muscle test, there was $2 / 5$ strength of the left shoulder flexor and abductor and $4 / 5$ strength of the left elbow flexor. Sensory examinations including pin prick and light touch were normal. DTRs in the biceps brachii and triceps brachii were also normal. In MRI of the cervical spine, there was no diffuse bulging

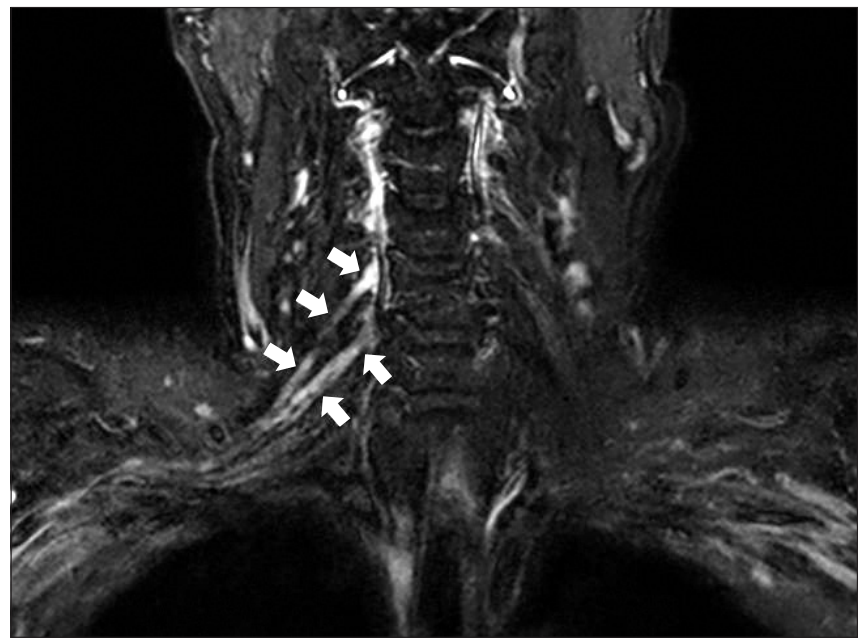

Fig. 3. The high signal intensity in the postganglionic right $\mathrm{C} 5$ and $\mathrm{C} 6$ root area (arrows) in the coronal T2 short-tau inversion recovery image. intervertebral disc or neural foraminal stenosis. Electrophysiologic studies including NCS and needle electromyography were compatible with left brachial plexopathy involving upper trunk.

MRN of the brachial plexus was performed at four weeks from the symptom onset (Fig. 4A, B). Using an 1.5-Tesla scanner (Achieva; Philips Medical Systems), coronal STIR images were recruited at TR/TE 6112/80. Gd-enhanced T1-weighted images were also recruited at TR/TE 584/10, in addition to conventional T1/T2-weighted images. An HSI lesion from the preganglionic C5 root to the level of cord in T2 STIR images and enhancement of the $\mathrm{C} 5$ root sheath in $\mathrm{T} 1 \mathrm{Gd}$ enhancement images were shown.

\section{DISCUSSION}

Shoulder pain is a fairly common symptom, and it has a number of differential diagnoses. If shoulder pain is accompanied by weakness, many other disorders including cervical radiculopathy are considered the primary causes with regard to the disease incidence [7]. NA has very low incidence rate and life-time prevalence, with shoulder pain and weakness as distinctive features. NA has different treatment methods from other disorders, such as cervical radiculopathy or rotator cuff disorder $[3,7]$. As such, correct diagnosis can provide the opportunity to avoid unnecessary surgical management and other spine manipulation.

For the diagnosis of HNA, diagnostic guidelines were developed by the European Charcot-Marie-Tooth Con-

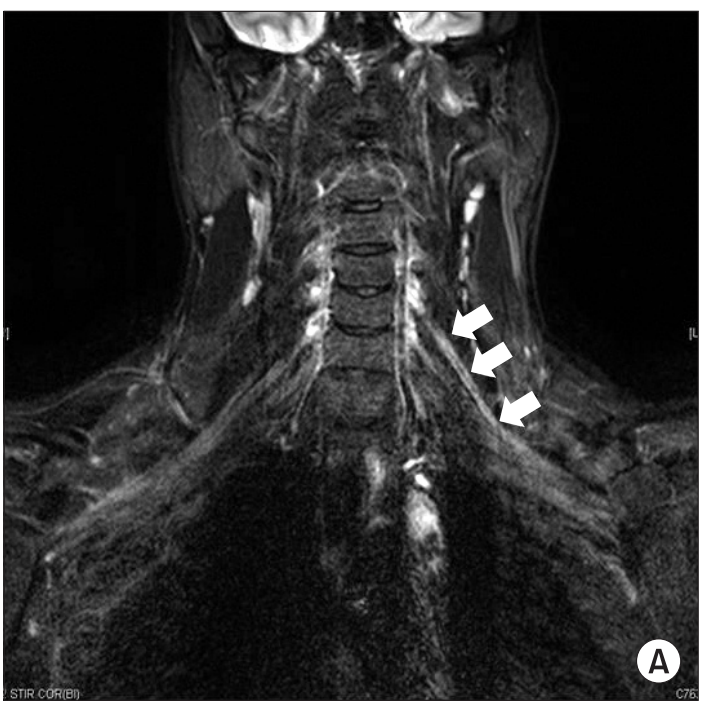

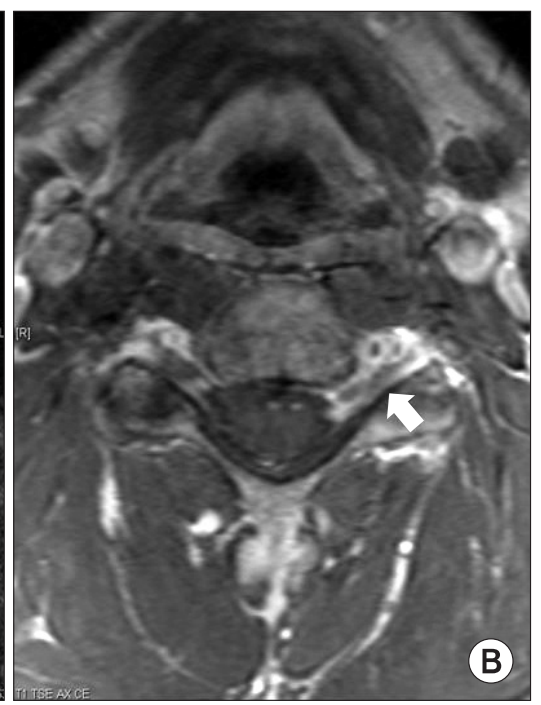

B)
Fig. 4. (A) The high signal intensity from pre- and post-ganglionic left C5 root to the area of the upper trunk formation in the coronal T2 short-tau inversion recovery image (arrows). (B) Enhancement of the $\mathrm{C} 5$ root sheath in the axial $\mathrm{T} 1$ gadolinium enhancement image (arrow). 
sortium. However, no definite standardized diagnostic criteria exist for INA. While the diagnostic guidelines for HNA have been used for selection and identification of INA patients in the majority of the literature, they have many limitations in differentiating it from other possible disorders that cause acute pain and weakness. It is challenging to differentiate INA from other neuromuscular disorders or rotator cuff disorders when patients present with pain and subsequent weakness.

Although MRI is a sensitive method for the evaluation of peripheral nerve pathologies, conventional MRI of the brachial plexus is not sensitive enough to identify pathologic changes in NA [8]. Recently, MRN, a new technique with a higher resolution, has been presented to be more sensitive for peripheral nervous system pathology $[4,5]$. In previous report, sensory changes in NA have been patchy and multifocal in distribution. It can appear in almost any type of involvement rather than corresponding to the site of plexus or nerve involvement [3]. However, NA can continuously involve the brachial plexus. In considering our MR neurographic images, there is a continuous lesion from the cervical root to the trunk or cord rather than a patchy involvement or multifocal distribution of the peripheral nerve. Sufficient further studies are required to find this appearance of nerve involvement in NA.

In this report of cases, we have compared the findings in MRN and electrodiagnostic study, which is one of the most important tools in the diagnosis of INA. The patient in Case 1 did not exhibit an abnormality in sensory NCS of the LABC nerves. Such did not fit with the findings in Fig. 1, which showed a brachial plexopathy involving upper trunk. However, in a previous report of 112 patients with NA, only $15 \%$ of the patients showed abnormal findings in sensory NCS of the LABC nerve [9]. Therefore, normal findings of sensory NCS in the LABC nerve may not preclude the diagnosis of INA, and it is required to consider the findings in MRN of the brachial plexus.

MRN demonstrated abnormal thickening and a hyperintensity lesion of the brachial plexus in the acute as well as chronic stage of NA $[4,5]$. These reports suggest that MRN be considered in the diagnosis of NA, as it has better imaging quality and may be superior to conventional MRI. According to the previous report for NA in subacute stage, conventional MRI showed atrophy and HSI with enhancement in the supraspinatus and infraspinatus [10].
However, these aspects were not sufficient to diagnose NA, because there were only indirect findings due to acute denervation following brachial plexopathy.

We present the first four cases of classic INA in subacute stage diagnosed with MRN, with thickened and/or enhanced brachial plexus as revealed with HSI. Although further prospective trials are required to determine its sensitivity and specificity as a new diagnostic method, MRN may contribute to confirming INA in subacute stage as well as in acute or chronic stage.

\section{CONFLICT OF INTEREST}

No potential conflict of interest relevant to this article was reported.

\section{REFERENCES}

1. van Alfen N, van der Werf SP, van Engelen BG. Longterm pain, fatigue, and impairment in neuralgic amyotrophy. Arch Phys Med Rehabil 2009;90:435-9.

2. Chance PF. Inherited focal, episodic neuropathies: hereditary neuropathy with liability to pressure palsies and hereditary neuralgic amyotrophy. Neuromolecular Med 2006;8:159-74.

3. van Alfen N, van Engelen BG. The clinical spectrum of neuralgic amyotrophy in 246 cases. Brain 2006;129(Pt 2):438-50.

4. Duman I, Guvenc I, Kalyon TA. Neuralgic amyotrophy, diagnosed with magnetic resonance neurography in acute stage: a case report and review of the literature. Neurologist 2007;13:219-21.

5. Sarikaya S, Sumer M, Ozdolap S, Erdem CZ. Magnetic resonance neurography diagnosed brachial plexitis: a case report. Arch Phys Med Rehabil 2005;86:1058-9.

6. van Alfen N. Clinical and pathophysiological concepts of neuralgic amyotrophy. Nat Rev Neurol 2011;7:31522.

7. Miller JD, Pruitt S, McDonald TJ. Acute brachial plexus neuritis: an uncommon cause of shoulder pain. Am Fam Physician 2000;62:2067-72.

8. Du R, Auguste KI, Chin CT, Engstrom JW, Weinstein PR. Magnetic resonance neurography for the evaluation of peripheral nerve, brachial plexus, and nerve root disorders. J Neurosurg 2010;112:362-71.

9. van Alfen N, Huisman WJ, Overeem S, van Engelen 
BG, Zwarts MJ. Sensory nerve conduction studies in neuralgic amyotrophy. Am J Phys Med Rehabil 2009;88:941-6.
10. Dill-Macky MJ, Song S, Silbert PL. Magnetic resonance imaging features of subacute idiopathic brachial neuritis. Australas Radiol 2000;44:98-100. 